ANNALES

POLONICI MATHEMATICI

$93.1(2008)$

\title{
On the mean-value property of superharmonic functions
}

\author{
by RoBert DaLmasso (Grenoble)
}

\begin{abstract}
We complement a previous result concerning a converse of the mean-value property for smooth superharmonic functions. The case of harmonic functions was treated by Kuran and an improvement was given by Armitage and Goldstein.
\end{abstract}

Recall that a function $u$ is harmonic (resp. superharmonic) on an open set $U \subset \mathbb{R}^{n}(n \geq 1)$ if $u \in C^{2}(U)$ and $\Delta u=0$ (resp. $\left.\Delta u \leq 0\right)$ on $U$. Denote by $H(U)$ the space of harmonic functions on $U$ and by $S H(U)$ the subset of $C^{2}(U)$ consisting of superharmonic functions on $U$. Notice that superharmonic functions are usually defined in a more general sense (see [5] and Remark 3).

If $A \subset \mathbb{R}^{n}$ is Lebesgue measurable, $L^{1}(A)$ denotes the space of Lebesgue integrable functions on $A$. If $A$ has finite measure we denote by $|A|$ the Lebesgue measure of $A$.

In [3] we proved the following theorem.

TheOREm 1. Let $\Omega \subset \mathbb{R}^{n}(n \geq 1)$ be a bounded open set. Suppose that there exists $x_{0} \in \Omega$ such that

$$
u\left(x_{0}\right) \geq \frac{1}{|\Omega|} \int_{\Omega} u(x) d x
$$

for every $u \in S H(\Omega) \cap L^{1}(\Omega) \backslash H(\Omega)$. Then $\Omega$ is a ball with center $x_{0}$.

Theorem 1 extends a result obtained by Epstein and Schiffer [4] for harmonic functions. The final step concerning harmonic functions was achieved by Kuran [6] who proved the following theorem.

ThEOREM 2. Let $\Omega \subset \mathbb{R}^{n}(n \geq 2)$ be a connected open set of finite measure. Suppose that there exists $x_{0} \in \Omega$ such that

$$
u\left(x_{0}\right)=\frac{1}{|\Omega|} \int_{\Omega} u(x) d x
$$

for every $u \in H(\Omega) \cap L^{1}(\Omega)$. Then $\Omega$ is a ball with center $x_{0}$.

2000 Mathematics Subject Classification: Primary 31B05.

Key words and phrases: superharmonic functions, mean-value property. 
REMARK 1. The proof of Theorem 2 given in [2] for $n=2$, which extends to $n \geq 2$, shows that the connectedness assumption is superfluous.

REMARK 2. The hypothesis of Theorem 2 can be weakened to require only that (1) holds for all positive harmonic functions that are integrable over $\Omega$ : see [1].

We first give a proof of the following result.

THEOREM 3. Let $\Omega \subset \mathbb{R}^{n}(n \geq 2)$ be an open set of finite measure. Suppose that there exists $x_{0} \in \Omega$ such that

$$
u\left(x_{0}\right) \geq \frac{1}{|\Omega|} \int_{\Omega} u(x) d x
$$

for every $u \in S H(\Omega) \cap L^{1}(\Omega) \backslash H(\Omega)$. Then $\Omega$ is a ball with center $x_{0}$.

Proof. We shall show that $\Omega$ satisfies the assumptions of Theorem 2 . Let $h \in H(\Omega) \cap L^{1}(\Omega)$. Let $y \in \mathbb{R}^{n} \backslash \Omega$ and $a_{n} \in(-n, 0)$. We define $v: \mathbb{R}^{n} \backslash\{y\} \rightarrow \mathbb{R}$ by

$$
v(x)=-\|x-y\|^{a_{n}}, \quad x \in \mathbb{R}^{n} \backslash\{y\} .
$$

We have $\Delta v<0$ in $\Omega$. Moreover, $v \in L^{1}(\Omega)$. Indeed, let $B(y, r)$ denote the open ball of fixed radius $r>0$. Clearly $v \in L^{1}(B(y, r))$. Since $\Omega$ has finite measure and $v$ is bounded on $\Omega \backslash B(y, r), v \in L^{1}(\Omega \backslash B(y, r))$ and the result follows. Now for $m \in \mathbb{N}^{\star}=\{1,2,3, \ldots\}$ we set

$$
u_{m}=h+\frac{1}{m} v .
$$

Then $u_{m} \in S H(\Omega) \cap L^{1}(\Omega) \backslash H(\Omega)$ and we have

$$
u_{m}\left(x_{0}\right) \geq \frac{1}{|\Omega|} \int_{\Omega} u_{m}(x) d x
$$

that is,

$$
h\left(x_{0}\right)+\frac{1}{m} v\left(x_{0}\right) \geq \frac{1}{|\Omega|} \int_{\Omega} h(x) d x+\frac{1}{m|\Omega|} \int_{\Omega} v(x) d x .
$$

Letting $m \rightarrow+\infty$ we obtain

$$
h\left(x_{0}\right) \geq \frac{1}{|\Omega|} \int_{\Omega} h(x) d x .
$$

As (3) holds for every $h \in H(\Omega) \cap L^{1}(\Omega)$, replacing $h$ by $-h$ in (3) we conclude that

$$
h\left(x_{0}\right)=\frac{1}{|\Omega|} \int_{\Omega} h(x) d x
$$

for all $h \in H(\Omega) \cap L^{1}(\Omega)$. Then Theorem 2 implies that $\Omega$ is a ball centered at $x_{0}$. 
Now we have the following theorem.

TheOREM 4. Let $\Omega \subset \mathbb{R}^{n}(n \geq 2)$ be an open set of finite measure. Suppose that, for all $y \in \partial \Omega$, there exists a sequence $\left(y_{j}\right)$ in $\mathbb{R}^{n} \backslash \bar{\Omega}$ such that $y_{j} \rightarrow y$ as $j \rightarrow+\infty$. If (2) holds for every positive $u \in S H(\Omega) \cap L^{1}(\Omega) \backslash$ $H(\Omega)$, then $\Omega$ is a ball with center $x_{0}$.

We shall need three lemmas.

Lemma 1. Let $\Omega \subset \mathbb{R}^{n}(n \geq 1)$ be an open set of finite measure. Suppose that (2) holds for all positive $u \in S H(\Omega) \cap L^{1}(\Omega) \backslash H(\Omega)$. Then (2) holds for all $u \in S H(\Omega) \cap L^{1}(\Omega) \backslash H(\Omega)$ that are bounded from below.

Proof. Let $u \in S H(\Omega) \cap L^{1}(\Omega) \backslash H(\Omega)$ be bounded from below. There exists $c \in \mathbb{R}$ such that $u>c$ on $\Omega$. Then $u-c \in S H(\Omega) \cap L^{1}(\Omega) \backslash H(\Omega)$ since $\Omega$ has finite measure, and $u-c$ is positive on $\Omega$. By hypothesis, $u-c$ has the mean value property (2). The constant function $c$ has the mean value property (1). Hence $u$ satisfies (2).

Lemma 2. Let $\Omega \subset \mathbb{R}^{n}(n \geq 1)$ be an open set of finite measure and let $\alpha \in(-n, 0)$.

(i) Suppose that there exists a sequence $\left(y_{j}\right)$ in $\mathbb{R}^{n} \backslash \bar{\Omega}$ such that $y_{j} \rightarrow$ $y \in \partial \Omega$ as $j \rightarrow+\infty$.

$$
\lim _{j \rightarrow+\infty} \int_{\Omega}\left\|x-y_{j}\right\|^{\alpha} d x=\int_{\Omega}\|x-y\|^{\alpha} d x .
$$

(ib) If $\left(b_{j}\right)$ is a sequence in $\mathbb{R}^{n}$ such that $b_{j} \rightarrow b$ as $j \rightarrow+\infty$, then

$$
\lim _{j \rightarrow+\infty} \int_{\Omega} b_{j} \cdot\left(x-y_{j}\right)\left\|x-y_{j}\right\|^{\alpha-1} d x=\int_{\Omega} b \cdot(x-y)\|x-y\|^{\alpha-1} d x .
$$

(ii) Suppose that there exists a sequence $\left(z_{j}\right)$ in $\mathbb{R}^{n} \backslash \bar{\Omega}$ such that $\left\|z_{j}\right\| \rightarrow+\infty$ as $j \rightarrow+\infty$. Then

$$
\lim _{j \rightarrow+\infty} \int_{\Omega}\left\|x-z_{j}\right\|^{\alpha} d x=0 .
$$

Proof. (i) Since the arguments are similar, we only prove (ia). Since $\Omega$ has finite measure the function $x \mapsto\left\|x-y_{j}\right\|^{\alpha}$ is in $L^{1}(\Omega)$ for every $j \in \mathbb{N}$ and we have seen in the proof of Theorem 3 that $x \mapsto\|x-y\|^{\alpha}$ is also in $L^{1}(\Omega)$. Let $r>0$ be fixed. There exists $j(r) \in \mathbb{N}$ such that $y_{j} \in B(y, r / 3)$ for all $j \geq j(r)$. For $j \geq j(r)$ we can write

$$
\int_{\Omega \cap B(y, r)}\left\|x-y_{j}\right\|^{\alpha} d x=\int_{\Omega \cap B\left(y_{j}, r / 3\right)}\left\|x-y_{j}\right\|^{\alpha} d x+\int_{\Omega \cap B(y, r) \backslash B\left(y_{j}, r / 3\right)}\left\|x-y_{j}\right\|^{\alpha} d x .
$$


We have

$$
\int_{\Omega \cap B\left(y_{j}, r / 3\right)}\left\|x-y_{j}\right\|^{\alpha} d x \leq C_{n} r^{n+\alpha}, \quad \int_{\Omega \cap B(y, r)}\|x-y\|^{\alpha} d x \leq C_{n} r^{n+\alpha}
$$

and

$$
\int_{\Omega \cap B(y, r) \backslash B\left(y_{j}, r / 3\right)}\left\|x-y_{j}\right\|^{\alpha} d x \leq(r / 3)^{\alpha}\left|\Omega \cap B(y, r) \backslash B\left(y_{j}, r / 3\right)\right| \leq C_{n} r^{n+\alpha},
$$

where $C_{n}>0$ is independent of $j$ and $r$. On the other hand, the Lebesgue dominated convergence theorem implies that

$$
\lim _{j \rightarrow+\infty} \int_{\Omega \backslash B(y, r)}\left\|x-y_{j}\right\|^{\alpha} d x=\int_{\Omega \backslash B(y, r)}\|x-y\|^{\alpha} d x .
$$

Now let $f_{j}(x)=\left\|x-y_{j}\right\|^{\alpha}-\|x-y\|^{\alpha}, x \in \Omega$. For $j \geq j(r)$ we write

$$
\begin{aligned}
\left|\int_{\Omega} f_{j}(x) d x\right| & \leq\left|\int_{\Omega \cap B(y, r)} f_{j}(x) d x\right|+\left|\int_{\Omega \backslash B(y, r)} f_{j}(x) d x\right| \\
& \leq 3 C_{n} r^{n+\alpha}+\left|\int_{\Omega \backslash B(y, r)} f_{j}(x) d x\right| .
\end{aligned}
$$

Let $\varepsilon>0$. Take $r>0$ such that $3 C_{n} r^{n+\alpha} \leq \varepsilon / 2$. By (4) there exists $j_{0} \geq j(r)$ such that

$$
\left|\int_{\Omega \backslash B(y, r)} f_{j}(x) d x\right| \leq \varepsilon / 2 \quad \forall j \geq j_{0},
$$

and (ia) follows.

(ii) For $k \in \mathbb{N}^{\star}$ define $\Omega_{k}=\Omega \backslash B(0, k)$. Let $k \in \mathbb{N}^{\star}$ and $r>0$ be fixed. We write

$$
\int_{\Omega}\left\|x-z_{j}\right\|^{\alpha} d x=\int_{\Omega \cap B(0, k)}\left\|x-z_{j}\right\|^{\alpha} d x+\int_{\Omega_{k}}\left\|x-z_{j}\right\|^{\alpha} d x .
$$

We have

$$
\int_{\Omega_{k} \backslash B\left(z_{j}, r\right)}\left\|x-z_{j}\right\|^{\alpha} d x \leq r^{\alpha}\left|\Omega_{k}\right|
$$

and

$$
\int_{\Omega_{k} \cap B\left(z_{j}, r\right)}\left\|x-z_{j}\right\|^{\alpha} d x \leq C_{n} r^{n+\alpha},
$$

where $C_{n}>0$ is independent of $j, k$ and $r$. Now let $\varepsilon>0$. Take $r>0$ such that $C_{n} r^{n+\alpha} \leq \varepsilon / 3$. Since $\left|\Omega_{k}\right| \rightarrow 0$ as $k \rightarrow+\infty$, there exists $k \in \mathbb{N}$ such that $r^{\alpha}\left|\Omega_{k}\right| \leq \varepsilon / 3$. By the Lebesgue dominated convergence theorem there exists $j_{0} \in \mathbb{N}$ such that

and (ii) follows.

$$
\int_{\Omega \cap B(0, k)}\left\|x-z_{j}\right\|^{\alpha} d x \leq \varepsilon / 3 \quad \forall j \geq j_{0},
$$


Lemma 3. In the setting of Theorem 4 there exists a sequence $\left(z_{j}\right)$ in $\mathbb{R}^{n} \backslash \bar{\Omega}$ such that $\left\|z_{j}\right\| \rightarrow+\infty$ as $j \rightarrow+\infty$.

Proof. Suppose first that $\partial \Omega$ is bounded. Since $\Omega$ has finite measure, we deduce that $\mathbb{R}^{n} \backslash \bar{\Omega}$ is unbounded and the lemma follows. Now, if $\partial \Omega$ is unbounded, there exists a sequence $\left(y_{j}\right)$ in $\partial \Omega$ such that $\left\|y_{j}\right\| \rightarrow+\infty$. By hypothesis, for each $j \in \mathbb{N}$ there exists $z_{j} \in\left(\mathbb{R}^{n} \backslash \bar{\Omega}\right) \cap B\left(y_{j}, 1\right)$. Clearly $\left\|z_{j}\right\| \rightarrow+\infty$.

Proof of Theorem 4. Since $\Omega$ has finite measure there exists a largest open ball $B$ centered at $x_{0}$ of radius $r$ which lies in $\Omega$. We will show that $\Omega=B$. There exists $y \in \partial \Omega \cap \partial B$ such that $\left\|y-x_{0}\right\|=r$. Let $\left(y_{j}\right)$ in $\mathbb{R}^{n} \backslash \bar{\Omega}$ be such that $y_{j} \rightarrow y$ as $j \rightarrow+\infty$ and let $\left(z_{j}\right)$ be as in Lemma 3 . Define

$$
\begin{gathered}
h(x)=r^{n-2}\left(\left\|x-x_{0}\right\|^{2}-r^{2}\right)\|x-y\|^{-n}, \quad x \in \mathbb{R}^{n} \backslash\{y\}, \\
h_{j}(x)=\left\|y_{j}-x_{0}\right\|^{n-2}\left(\left\|x-x_{0}\right\|^{2}-\left\|y_{j}-x_{0}\right\|^{2}\right)\left\|x-y_{j}\right\|^{-n}, \quad x \in \mathbb{R}^{n} \backslash\left\{y_{j}\right\},
\end{gathered}
$$

and

$$
v_{j}(x)=-\left\|x-z_{j}\right\|^{a_{n}}, \quad x \in \mathbb{R}^{n} \backslash\left\{z_{j}\right\},
$$

where $a_{n} \in(-n, 0)$. Clearly $h \in H\left(\mathbb{R}^{n} \backslash\{y\}\right), h_{j} \in H\left(\mathbb{R}^{n} \backslash\left\{y_{j}\right\}\right)$ and $\Delta v_{j}<0$ in $\Omega$. Moreover $h, h_{j}$ and $v_{j}$ are in $L^{1}(\Omega), h\left(x_{0}\right)=-1$ and $h>0$ on $\mathbb{R}^{n} \backslash \bar{B}$. Let $u_{j}=1+h_{j}+v_{j}$. Then $u_{j} \in S H(\Omega) \cap L^{1}(\Omega) \backslash H(\Omega)$ and $u_{j}$ is bounded from below on $\Omega$ for every $j$. Therefore Lemma 1 implies that

$$
u_{j}\left(x_{0}\right) \geq \frac{1}{|\Omega|} \int_{\Omega} u_{j}(x) d x \quad \forall j \in \mathbb{N} .
$$

By Lemma 2 we can let $j \rightarrow+\infty$ in (5) to obtain

$$
1+h\left(x_{0}\right) \geq \frac{1}{|\Omega|} \int_{\Omega}(1+h(x)) d x .
$$

Since $1+h \in H(\Omega) \cap L^{1}(\Omega)$ we have

$$
0=1+h\left(x_{0}\right)=\int_{B}(1+h(x)) d x
$$

Now with the help of (6) and (7) we can write

$$
\begin{aligned}
0 & \geq \frac{1}{|\Omega|} \int_{\Omega}(1+h(x)) d x \\
& =\frac{1}{|\Omega|} \int_{\Omega \backslash B}(1+h(x)) d x+\frac{1}{|\Omega|} \int_{B}(1+h(x)) d x \\
& =\frac{1}{|\Omega|} \int_{\Omega \backslash B}(1+h(x)) d x \geq \frac{|\Omega \backslash B|}{|\Omega|} \geq \frac{|\Omega \backslash \bar{B}|}{|\Omega|} .
\end{aligned}
$$


This implies that $|\Omega \backslash \bar{B}|=0$. Then the open set $\Omega \backslash \bar{B}$ must be empty, hence $\Omega \subset \bar{B}$. Since $\Omega$ is open and $B \subset \Omega \subset \bar{B}$, we deduce that $\Omega=B$.

REMARK 3. The assumption in Theorem 2 imposes a certain geometric restriction on the open set $\Omega$. We give an example that shows that this hypothesis cannot be omitted completely. Let $\Omega=B \backslash\{x\}$ where $B$ denotes an open ball centered at the origin in $\mathbb{R}^{n}(n \geq 2)$ and $x \in B \backslash\{0\}$. We claim that if $u \in L^{1}(\Omega)$ is a positive superharmonic function on $\Omega$, then (2) holds. Indeed such a function $u$ has a (unique) superharmonic extension $v$ on $B$ (see $[5$, Theorem 7.7, p. 130]). Then we have

$$
u(0)=v(0) \geq \frac{1}{|B|} \int_{B} v(y) d y=\frac{1}{|\Omega|} \int_{\Omega} u(y) d y .
$$

In fact, using Theorem 7.7 in [5], we can take $\Omega=B \backslash Z$ where $Z$ is a relatively closed polar subset of $B$ such that $0 \notin Z$. For instance in $\mathbb{R}^{3}, Z$ could be a line segment (see [5, Example 4, p. 127]).

\section{References}

[1] D. H. Armitage and M. Goldstein, The volume mean-value property of harmonic functions, Complex Variables 13 (1990), 185-193.

[2] R. B. Burckel, Three secrets about harmonic functions, Amer. Math. Monthly 104 (1997), 52-56.

[3] R. Dalmasso, On the mean value property of superharmonic and subharmonic functions, Int. J. Math. Math. Sci. 2 (2006), 1-3.

[4] B. Epstein and M. Schiffer, On the mean-value property of harmonic functions, J. Anal. Math. 14 (1965), 109-111.

[5] L. L. Helms, Introduction to Potential Theory, Wiley-Interscience, New York, 1969.

[6] Ü. Kuran, On the mean-value property of harmonic functions, Bull. London Math. Soc. 4 (1972), 311-312.

Laboratoire Jean Kuntzmann

Equipe EDP

Tour IRMA - BP 53

38041 Grenoble Cedex 9, France

E-mail: robert.dalmasso@imag.fr

Received 6.9.2007

and in final form 8.1.2008 